Pacific Journal of Mathematics

SIMPLE SEPARABLE GRAPHS 


\section{SIMPLE SEPARABLE GRAPHS}

\section{R. H. JOHNSON}

The relation between the structure of a graph and the degrees of its vertices is a problem that has long occupied graph theorists in one form or another. If the degrees of the vertices of a graph are arranged in nonincreasing order the sequence obtained is the degree sequence of the graph. Thus the above problem is often formulated as "how does the degree sequence affect the structure of the graph?" One approach is to discover which graphs are determined up to isomorphism by their degree sequence. Following Harary, these latter graphs and their degree sequences are called simple. In simple graphs the effect of the degree sequence on structure is, in a good sense, isolated. In this paper all simple graphs which are not blocks are determined.

The paper will proceed as follows. In §II elementary but essential properties of simple graphs are given (e.g., simple regular graphs determined). Simple trees are listed in \$III. The latter results are then used in §IV to find all simple graphs which are not separable.

Before proceeding it is worth noting that Hakimi in [3] and Senior in [6] have given a relatively complete treatment of simple multi-graphs and simple psuedo-graphs. By the term 'graph' we have denoted, and shall continue to do so, the 'ordinary' graph of [1] — indeed all the terminology of this paper is that of [1]. Since any graph may be considered to be multi-graph or a psuedo-graph but not conversely it should not came as a surprise that a simple graph may not be a simple multi-graph or a simple psuedo-graph. That the class of simple graphs is far more rich and complex than either of the latter is seen by comparing the results obtained below with those found in either [3] or [6].

The basic concept used to carry out the above project is the concept of 'transfer' or 'degree preserving' transformation.

DEFINITION. Let $G$ be a graph and $x, y, u, v$ be four distinct points of $V G$ (the vertex set of $G$ ) such that $x y, u v \in E G$ (the edge set of $G$ ) but $x u, y v \notin E G$. A transfer $t$ of $G$ is the replacement of the edges $x y$ and $u v$ by $x u$ and $y v$. The graph so obtained is denoted by $t G$.

It is immediate that $G$ and $t G$ have the same degree sequence. But the converse is also true. We quote the following result of [2]. 
THEOREM 1.1. If graphs $G$ and $H$ have the same degree sequence then there exists a finite number of transfers $t_{1}, \cdots, t_{r}$ such that

$$
G \cong t_{1} \circ \cdots \circ t_{r} H
$$

2. The results of this section are essential but easy. For the sake of completeness all proofs are given - at the risk of being wearisome.

Proposition 2.1. A graph $G$ is simple if and only if for each transfer $t$ of $G$ we have $G \cong t G$.

Proof. This follows directly from 1.1 .

Proposition 2.2. A graph $G$ is simple if and only if $G^{c}$ (the complement of $G$ ) is simple.

Proof. First note that if $f$ is an isomorphism of graphs $G$ and $H$, then $f$ is also an isomorphism of $G^{c}$ and $H^{c}$. Let $G$ be simple and let $H^{\prime}$ belong to the same sequence as $G^{c}$. Then $\left(H^{\prime}\right)^{c}$ belongs to the same sequence as $G$ does so that $G \cong\left(H^{\prime}\right)^{c}$. Hence by the above $G^{c} \cong H^{\prime}$ so that $G^{c}$ simple. A similar argument shows that $G^{c}$ simple implies that $G$ is.

If $S$ is a sequence, $|S|$ denotes the number of realizations of $S$.

Proposition 2.3. $S=\left(d_{1}, \cdots, d_{p}\right)$ is a graphical sequence if and only if $S^{\prime}=\left(p-1-d_{p}, \cdots, p-1-d_{1}\right)$ is. Moreover, $|S|=\left|S^{\prime}\right|$.

Proof. One need only note that $G$ belongs to $\left(d_{1}, \cdots, d_{p}\right)$ if and only if $G^{c}$ belongs to $\left(p-1-d_{p}, \cdots, p-1-d_{1}\right)$ and use above proposition.

Proposition 2.4. The sequences $\left(d_{1}, \cdots, d_{p}\right),\left(p, d_{1}+1, \cdots, d_{p}+1\right)$ and $\left(d_{1}, \cdots, d_{p}, 0\right)$ have the same number of realizations.

Pröof. It is clear that $\left|\left(d_{1}, \cdots, d_{p}\right)\right|=\left|\left(d_{1}, \cdots, d_{p}, 0\right)\right|$. Applying 2.3 twice we have

$$
\begin{aligned}
\left|\left(d_{1}, \cdots, d_{p}\right)\right| & =\left|\left(p-1-d_{1}, \cdots, p-1-d_{p}\right)\right| \\
& =\left|\left(p-1-d_{1}, \cdots, p-1-d_{p}, 0\right)\right| \\
& =\left|\left(p, d_{1}+1, \cdots, d_{p}+1\right)\right|
\end{aligned}
$$

REMARK. It should be clear that any realization of $\left(p, d_{1}+\right.$ $\left.1, \cdots, d_{p}+1\right)$, if there are any, can be obtained from a realization of 
$\left(d_{1}, \cdots, d_{p}\right)$ by adding a new point adjacent to all other points of the latter realization.

CoROllary 2.5. The sequences $\left(d_{1}, \cdots, d_{p}\right),\left(p, d_{1}+1, \cdots, d_{p}+1\right)$, $\left(d_{1}, \cdots, d_{p, 0}\right)$ are either all simple or all not simple.

REMARK. We now give some examples of simple graphs and sequences. First note that $K_{p}$ and the star graph $K_{1, p}$ are simple because no elememtary transfers can be made (defined). We define a $\theta_{p}$ graph to be a realization of the sequence $(p, p, 2, \cdots, 2)$ of length $p+1$. Since a $\theta_{p}$ graph is obtained from a $K_{1, p}$ by adding a point adjacent to all others it follows from 2.4 that $\theta_{p}$ is simple. A cycle $C_{p}$ is simple if and only if $p \leqq 5$ since if $p \geqq 6$ a single transfer can be made on $C_{p}$ to create a disconnected graph. One can verify that all graphs on four or less points are simple. The next proposition guarantees the existence of many examples of simple graphs.

Proposition 2.6. For any positive integer $p$ and any integer $q$ such that $0 \leqq q \leqq p(p-1) / 2$ there is a simple $(p, q)$ graph.

Proof. The proof is by induction on $p$. Since the trivial graph is simple, the result is true for $p=1$. Assume the proposition is true for $p \leqq k$, and let $q$ be an integer such that

$$
0 \leqq q \leqq(k+1) k / 2 \text {. }
$$

If $q \leqq k(k-1) / 2$ then the result follows from the induction hypothesis since there is a simple $(k, q)$ graph from which a simple $(k+1, q)$ graph is obtained by adding a point of degree zero (Corollary 2.5).

On the other hand if $q$ satisfies

$$
k(k-1) / 2 \leqq q
$$

then $q=l+k(k-1) / 2$ where $0 \leqq l \leqq k$. To get the desired simple graph we adjoin a new point to any $l$ points of $K_{k}$. The graph so obtained is simple because its complement is the union of $K_{1, k-l}$ and trivial graphs.

The next result goes in the opposite direction.

Proposition 2.7. Let $p ; q$ be positive integers such that $p \geqq 5$ and $4 \leqq q \leqq p(p-1) / 2-4$. There is $a(p, q)$ graph which is not simple.

Proof. For $p=5$ the possible values for $q$ are four, five and six. Each pair of nonisomorphic graphs given below realizes the given sequence. 
(a) $(2,2,2,1,1) G_{4}=K_{3} \cup K_{2}, H_{4}=P_{5}$
(b) $(3,2,2,2,1) G_{5}=\square \square, H_{5}=\square$
(c) $(3,3,2,2,2)$
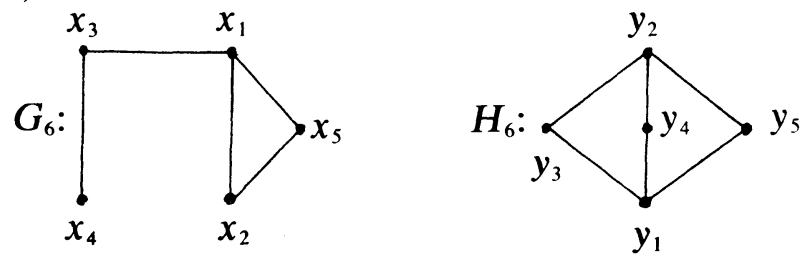

Note that each of $G_{4}, G_{5}, G_{6}$ have a triangle while none of $H_{4}, H_{5}, H_{6}$ does.

For $p>5$ and $j$ such that $4 \leqq j \leqq p(p-1) / 2-4$ we construct $(p, j)$ graphs $G_{j}$ and $H_{j}$ as follows. For $j=4,5,6$ we form the union of the above $G_{j}$ and $H_{j}$ with trivial graphs to obtain the required results. For $j>6$ let $V G_{j}=\left\{x_{1}, \cdots, x_{p}\right\}, V H_{j}=\left\{y_{1}, \cdots, y_{p}\right\}$, let $x_{1}, x_{2}, \cdots, x_{5}$ be related exactly as in $G_{6}$ above. Then adjoin $x_{6}$ to $x_{1}, \cdots, x_{5}$ in turn and $x_{7}$ to $x_{1}, \cdots, x_{6}$ in turn and so on until we have $j$ edges. The graph so obtained is $G_{j}$. Let $H_{j}$ be constructed from $H_{6}$ (above) in the same fashion. It is easily verified that (i) $G_{j}$ and $H_{j}$ belong to the same sequence, and (ii) at each stage of the construction there is a difference of at least one triangle between the two graphs. This yields the proposition.

The next sequence of Lemmas leads to a theorem giving necessary and sufficient conditions for a regular graph to be simple.

LemMa 2.8. Let $p$ be a positive integer $p \geqq 7, p=2 m+1$.

(a) If $m$ is odd, the sequence of length $p,(m+1, \cdots, m+1)$ is not simple.

(b) If $m$ even the sequence of length $p,(m, \cdots, m)$ is not simple.

Proof. Suppose $m$ is odd. On $X=\left\{x_{1}, \cdots, x_{m+1}\right\}$ and $Y=$ $\left\{y_{1}, \cdots, y_{m}\right\}$ construct a complete bipartite graph - let the edge set be $\left\{\left\{x_{i} y_{j}\right\} \mid 1 \leqq i \leqq m+1,1 \leqq j \leqq m\right\}$. Next add the edges $x_{1} x_{2}, x_{3} x_{4}, \cdots, x_{m} x_{m+1}$ - which is possible since $m$ is odd - and call the resulting graph $G$. Let $t$ be the transfer of $x_{1} y_{1}$ and $x_{m+1} y_{m}$ for $x_{1} x_{m+1}$ and $y_{1} y_{m}$. We compare the number of triangles in $G$ and $t G$. Since a bipartite graph has no triangles, the addition of the edges $x_{i} x_{i+1}, 1 \leqq i \leqq m$ introduces the only triangles into $G$. Clearly each such $x_{i} x_{i+1}$ lies on precisely $m$ triangles since we started with a complete bipartite graph. Hence, $G$ has a total of $(m+1) m / 2$ triangles. By similar reasoning $t G$ has $\left\{\frac{1}{2}(m+1) m-2\right\}+(m-2)+(m-1)$ triangles. This proves (a).

Assume now $m$ is even. As above construct the complete bipartite graph on the point sets $\left\{x_{1}, \cdots, x_{m}\right\}$ and $\left\{y_{1}, \cdots, y_{m}\right\}$. Then (i) delete the edges $x_{1} y_{1}, \cdots, x_{m} y_{m}$, (ii) add the edges $x_{1} x_{2}, x_{3} x_{4}, \cdots, x_{m-1} x_{m}$ (possible 
since $m$ even) and (iii) add a point $y$ adjacent to $y_{1}, \cdots, y_{m}$. Call the resulting graph $G$ and note that $G$ belongs to the sequence $(m, \cdots, m)$ of length $2 m+1$. Let $t$ be the transfer of $x_{2} y_{2}$ and $x_{m-1} y_{m}$ for $x_{2} x_{m-1}$ and $y_{1} y_{m}$. Counting triangles we have $G$ with $m(m-2) / 2$ and $t(G)$ with $m(m-2) / 2+2(m-4)+1$. This proves (b).

LEMMA 2.9. For $p=2 m \geqq 6$ the sequence $(m, \cdots, m)$ of length $p$ is not simple.

Proof. $K_{m, m}$ has no triangles but clearly an elementary transfer of it does.

Theorem of Erdos and Gallai. Let $S=\left(d_{1}, \cdots, d_{p}\right)$ be a sequence of nonnegative integers such that $d_{1}+\cdots+d_{p}$ is even. Then $S$ is graphical if and only if for each positive integer $r, 1 \leqq r \leqq p-1$

$$
\text { (*) } d_{1}+\cdots+d_{r} \leqq r(r-1)+\min \left\{r, d_{r+1}\right\}+\cdots+\min \left\{r, d_{p}\right\} \text {. }
$$

This result was proven by Erdos and Gallai and can be found in Harary ([4, p. 59-62]).

LemMA 2.10. For $p, m$, positive integers, $0 \leqq m \leqq p-1$ the se. quence $S=(m, \cdots, m)$ of length $p$ is graphical provided $m$ is even when $p$ is odd. At least one realization of $S$ is connected when $m \geqq 2$.

Proof. If $r$ is less than $m$ then (*) above becomes, for $S$,

$$
m \cdot r \leqq r(r-1)+m(p-r) .
$$

If $r$ is greater than or equal to $m$ then it can be verified that $(*)$ becomes

$$
r \cdot m \leqq r(r-1)+r(p-r)=r(p-1) .
$$

Hence, in either case $(*)$ is satisfied so that $S$ must be graphical. Finally, if $m \geqq 2$, it follows immediately from Proposition 1.6 of [5] that $S$ has at least one connected realization.

PROPOSITION 2.11. Let $p, r$ be positive integers such that $p \geqq 6$ and $2 \leqq r \leqq[p / 2]-1$. Then the sequence $S=(r, \cdots, r)$ of length $p$ has both a connected and disconnected realization, provided $m$ is even when $p$ is odd.

Proof. It suffices to exhibit the disconnected realization. If $p=6$ the only permissible value for $r$ is 2 and $K_{3} \cup K_{3}$ is the required graph. Now assume $p \geqq 7$. There are two cases. (a) $p=2 m$. Here $[p / 2]-1=m-1$. If $r=m-1, K_{m} \cup K_{m}$ suffices. For $r, 2 \leqq r \leqq$ 
$m-2$ we have two sub-cases. If $m$ is even, the union of two regular graphs of degree $r$ will do. If $m$ is odd, then the union of two regular graphs of degree $r$ on $m+1$ and $m-1$ points will do.

(b) $p=2 m+1=m+(m+1)$. Here $[p / 2]-1=m-1$. Since $p$ is odd $r$ can only assume even values and thus regardless of the parity of $m$ one can construct the desired graph by taking the union of regular graphs of degree $r$ on $m+1$ and $m$ points.

THEOREM 2.12. If $G$ is a regular graph of degree $r$ on p points, then $G$ is simple if and only if $r \in\{0,1, p-2, p-1\}$.

Proof. Since $G$ is simple if and only if $G^{c}$ is the result follows from 2.8, 2.9, 2.10 and 2.11.

3. Simple trees. In this section the simple graphs which are trees are characterized. To this end we define the 'Giap' graphs.

Definition. Let $p$ be a positive integer, $p \geqq 2$, and let $S=$ $(m, n, 1, \cdots, 1)$ be a sequence of length $p$ where $p=m+n$. Then any realization of $S$ is a Giap graph or an $[m, n] G$, or an $[m, n]$-Giap graph.

\section{LEMMA 3.1. Giap graphs are simple.}

Proof. Let $p$ be a positive integer, $p \geqq 2$ and let $S=$ $(m, n, 1, \cdots, 1), p=m+n$. One realization $G$ of $S$ can be defined as follows. Let $V(G)=\left\{x_{1}, \cdots, x_{p}\right\}$, where $\operatorname{deg} x_{1}=m, \quad \operatorname{deg} x_{2}=$ $n, x_{1} x_{2} \in E(G), x_{1}$ is adjacent to $m-1$ points of degree one and $x_{2}$ is adjacent to $n-1$ points of degree one. $G$ can be constructed by connecting a $K_{1, m-1}$ with a $K_{1, n-1}$ by an edge at their points of maximal degree. The only transfer that is defined on $G$ is of the following type. Let $y_{1}, y_{2} \in V(G), \quad y_{1} \neq x_{2}, \quad y_{2} \neq x_{1} \quad$ with $\quad y_{1} x_{1} \in E G, \quad y_{2} x_{2} \in$ $E G$. Then the transfer $t$ of $y_{1} x_{1}$ and $y_{2} x_{2}$ for $y_{2} x_{1}$ and $y_{1} x_{2}$ yields a graph isomorphic to $G$, and is the only possible kind of transfer since $x_{1} x_{2} \in E G$. (Note: The transfer $t$ amounts to interchanging points of degree one adjacent to $x_{1}$ and $x_{2}$.)

THEOREM 3.2. A tree is simple if and only if it is a Giap graph.

Proof. Since Lemma 3.1 says Giap graphs are simple and the proof of 3.1 shows that Giap graphs are trees, sufficiency is clear.

Now let $T$ be a simple tree. We first show that $T$ has no path of length four and hence has no path of length greater than three. Suppose the contrary i.e., there exist $y_{0}, y_{1}, \cdots, y_{4} \in V T$ for which $y_{0} y_{1} y_{2} y_{3} y_{4}$ is a path in $T$. Since $T$ is a tree neither $y_{0} y_{4}$ nor $y_{1} y_{3}$ 
are in ET so that the transfer $t$ of $y_{0} y_{1}$ and $y_{3} y_{4}$ for $y_{0} y_{4}$ and $y_{1} y_{3}$ is defined and $t T \neq T$ since $t T$ has a triangle. Hence, $T$ simple implies that $d T \leqq 3$, i.e., the diameter of $T$ is less than or equal to 3 .

Now let $x \in V T$ with $\operatorname{deg} x>1$. If each point of $N_{x}$ has degree one, then $T$ is a star graph, i.e., [ $p, 1]$-Giap graph. If $N_{x}$ has two points $x^{\prime}, x^{\prime \prime}$ of degree greater than one, then these exist $a^{\prime}, a^{\prime \prime} \in V T$ with $a^{\prime} x^{\prime}$, $a^{\prime \prime} x^{\prime \prime} \in E T, a^{\prime} \neq x, a^{\prime \prime} \neq x$. Since $T$ has no cycles $a^{\prime} \neq a^{\prime \prime}$ and thus $a^{\prime} x^{\prime} x x^{\prime \prime} a^{\prime \prime}$ is a path of length four, contradicting the above. Hence, $N_{x}$ has at most one point, say $x^{\prime}$, of degree greater than one. Reasoning as above it follows that $N_{x^{\prime}}$ has only one point of degree greater than one which must be $x$. Since $T$ is connected, VT consists of $x, x^{\prime}$ and points of degree one adjacent to either $x$ of $x^{\prime}$. Hence, $T$ is a Giap graph.

Observe that the above argument also shows that if $T$ is a tree with $d T \leqq 3$, then $T$ is a Giap graph. That is, the simplicity of $T$ was used to derive $d T \leqq 3$ and then from the latter the structure of $T$ was derived. This yields the following corollary.

Corollary 3.3. Let $T$ be a tree. Tis simple if and only if $d T \leqq 3$.

\section{Simple separable graphs.}

Proposition 4.1. Let $G$ be a disconnected graph with nontrivial components. $G$ is simple if and only if

$$
\text { (*) } G=s K_{2} \cup t[m, n] G
$$

where $s$ is a nonnegative integer, $t \in\{0,1\}$ and $s \neq 0$ implies either $m=1$ or $n=1$.

Proof. It is easy to verify that a graph of the form (*) is simple. Conversely assume that $G$ is simple and disconnected. First it is shown that no component of $G$ (of which there are at least two) contains a cycle. Let $C$ be a component and suppose to the contrary that $C$ contains a cycle and $x, y \in V C$ with $x y$ on the cycle. Let $x^{\prime}, y^{\prime} \in V C^{\prime}$, where $C^{\prime}$ is another component of $G$, with $x^{\prime} y^{\prime} \in$ $E C^{\prime}$. The transfer $t$ of $x y$ and $x^{\prime} y^{\prime}$ for $x x^{\prime}$ and $y y^{\prime}$ is defined and $G \neq t G$ since $t G$ has one fewer component than $G$. This latter contradicts the fact that $G$ is simple. It now follows that $G$ is a forest, and since each component of $G$ must be simple, the component of $G$ must be Giap graphs or trivial graphs.

Now suppose that $[n, m] G$ and $[k, l] G$ are components of $G$. We show this is impossible unless at least three of the four numbers $n, m, k, l$, are one. If $n \geqq 2, k \geqq 2$ and $m=l=1$, then a transfer can be yielding a $K_{1, n}$ and $K_{1, m}$. If $n \geqq 2, k \geqq 2$, then a transfer exists which 
creates an additional $K_{2}$. Hence in either case a transfer exists which creates different components so that the graph cannot be simple. This gives the result.

Remark. Since $G$ is simple if and only if $G^{c}$ is simple, 4.1 yields at once a criterion for a simple graph to have a disconnected complement.

The above characterizes disconnected simple graphs. We now turn to connected simple graphs with cut points, or separable simple graphs. For the present we consider graphs without vertices of degree one (without pendant vertices).

Proposition 4.2. Let $G$ be a connected graph without pendant vertices. If $G$ has two or more cut points, then $G$ cannot be simple.

Proof. By Lemma 1.9 of [5] we conclude that such a graph $G$ belongs to a sequence $S$ which contains another graph with at most one cut point. Thus $G$ cannot be simple.

The above proposition shows that to characterize connected simple graphs which are not blocks we need consider only those connected graphs with one cut point.

Definition. Let $T$ be a tree on $p$ points. A 1-cone of $T$ is a graph obtained from $T$ by adding a point to $T$ which is adjacent to at least the points of degree one of $T$, and perhaps other points of $T$.

Lemma 4.3. Let $T$ be a tree on two or more points. If $G$ is a 1-cone of $T$, then $G$ is a block.

Proof. Let $x, y \in V T$. It is clear that $x$ and $y$ belong to a path in $T$ where end points are points of degree one. In $G$ these latter points are adjacent to the same point and hence $x$ and $y$ lie on a cycle. If $z \in V G-V T$, then one can show in a similar fashion that for any $x \in V T, z$ and $x$ lie on a cycle also, yielding the result.

Lemma 4.4. Let T be a tree which is not simple. Then any 1-cone of $T$ is not simple.

Proof. If $T$ is not simple, it follows by Corollary 3.3 that there is a path $P$ of length greater than or equal to four. As above one may assume that the end points $x, y$ have degree one. If the path is $x z_{1} \cdots z_{r} y$, then the transfer $t$ of $x z_{1}$ and $z_{r} y$ for $z_{1} z_{r}$ and $x y$ is defined since $z_{1} \neq z_{r}$ and $z_{1} z_{1} \notin E T$. Hence $t T$ has a component $K_{2}$. If $G$ is any 1-cone of $T$, the same transfer $t$ is defined on $G$ and $G \neq t G$ since 
$t G$ has a $K_{3}$ as a block and by Lemma $4.3, G$ is itself a block.

Lemma 4.5. Let $T$ be a Giap graph with $T \neq P_{i}, i=1,2,3$. Then a 1 -cone $G$ of $T$ is simple if and only if the point of $V G-V T$ is adjacent to each point of VT. (Note that $P_{n}$ denotes a path of length $n$.)

Proof. Let $T$ be a Giap graph and $z \in V G-V T$ where $G$ is a 1 -cone of $T$. If $z$ adjacent to every point of $V T$, it follows from Proposition 2.4 that $G$ is simple.

Now suppose $G$ is simple. If $T=K_{2}=P_{2}$, then $G=K_{3}$ and $z$ is adjacent to each point of $K_{2}$. Thus assume $T$ is an $[n, m] G$ different from $P_{2}, P_{3}, P_{4}$. Then at least one of $n$ and $m$, say $n$ is greater than two. The new point $z$ has degree greater than or equal to $\max \{n, m\}$.

Let $n=\max \{n, m\}$ and suppose $z$ is not adjacent to either $x_{1}$ or $x_{2}$ in $T$ where $\operatorname{deg} x_{1}=n$, deg $x_{2}=m$ and $x_{1} x_{2} \in E T$. First assume $m=$ 1. Then $z x_{1} \notin E G$ but $z x_{2} \in E G$. How $n \geqq 2$ implies there is an $x_{3}$ such that $x_{1} x_{3}, z x_{3} \in E G$ and $x_{1} x_{2} \in E G$. The transfer $t$ of $x_{2} x_{1}$ and $x_{3} z$ for $x_{2} x_{3}$ and $x_{1} z$ changes the adjacency relations of $G$ i.e., $t G$ has more points of highest degree adjacent. Hence $t G \not \equiv G$.

Next assume $\operatorname{deg} x_{2}=m>1$, and let $x_{4} \in V T$ such $x_{2} x_{4} \in E T$. Since deg $x_{4}=1$, we have $z x_{4} \in E G$ and $x_{1} x_{4} \notin E(C)$. If $z$ is not adjacent to $x_{1}$, then the transfer $t$ of $z x_{3}$ and $x_{1} x_{2}$ for $z x_{1}$ and $x_{2} x_{3}$ again changes adjacency relations. Finally suppose that $z x_{1} \in E G$, but $z x_{2} \notin E G$. If $\operatorname{deg} x_{2}$ is greater than two, we can transfer as per above to get $z$ adjacent to $x_{2}$, leaving $z$ adjacent to $x_{1}$. If deg $x_{2}=2$, then the transfer of $z x_{1}$ and $x_{2} x_{4}$ for $x_{1} x_{4}$ and $z x_{2}$ yields a graph in which the points of highest degree $z, x_{1}$ are not adjacent. Thus in any case $G$ is not simple. This proves the lemma.

LemMA 4.6. If $T=P_{i}, i=1,2,3$ then any 1-cone of $T$ is simple.

Proof. The proof follows from looking at possible cases.

Definition. Let $G_{1}, G_{2}$ be graphs. Let $V G_{1} \cap V G_{2}=\phi$ and $x_{1}, y_{1}$ be points of maximal degree of $V G_{1}, V G_{2}$ respectively. By $G_{1} * G_{2}$ we mean the graph obtained by identifying $x_{1}$ and $y_{1}$ assuming that the construction is independent of the points of maximal degree chosen. Otherwise, $G_{1} * G_{2}$ is not defined.

LemMA 4.7. Let $S_{1}$ be the set of points of degree one in a tree $T_{1}$. Let $G_{1}$ be a cone of $T_{1}$. Let $G_{2}$ be a cone of a tree $T_{2}$. If $T_{1}$ is not a star graph, $\left|V T_{1}-S_{1}\right| \geqq 2$, then $G_{1} * G_{2}$ is defined and not simple.

Proof. If $G_{1}$ is not simple, then neither is $G_{1} * G_{2}$. Hence, assume $G_{1}$ is simple. 
Case 1. $T_{2}=K_{2}$. Here one block of $G_{1} * G_{2}$ is a triangle and $T_{1}$ is, by assumption and Lemma 4.4 , an $[n, m] G$ with $n \geqq 2, m \geqq 2$. Let $x y$ be the edge of $[n, m] G$ with $\operatorname{deg} x=n, \operatorname{deg} y=m$ and let $x_{1} y_{1}$ be the edge of $K_{2}$. Then the transfer of $x y$ and $x_{1} y_{1}$ for $x x_{1}$ yields a graph without a triangle as a block.

Case 2. $T_{2} \neq K_{2}$. Again by assumption $T_{1}$ cannot be a $K_{2}$ so that $G * G_{2}$ does not have a triangular block. Let $y_{1} \in V T_{1}, z_{1} \in V T_{2}$ with $\operatorname{deg} y_{1}=\operatorname{deg} z_{1}=1$ and let $y \in V T_{1}, z \in V T_{2}$ such that $y y_{1} \in E T_{1}, z z_{1} \in$ $E T_{2}$. Then the transfer of $y_{1} y$ and $z z_{1}$ for $y z$ and $y_{1} z_{1}$ creates a block which is a triangle. Hence in either case $G_{1} * G_{2}$ is not simple.

It is easy to verify that $G_{1} * G_{2}$ is defined whenever $G_{1}$ and $G_{2}$ are 1-cones.

Lemma 4.9. If $T_{1}, \cdots, T_{l}$ are trees having $G_{1}, \cdots, G_{l}$ as 1 -cones and $G_{1} * \cdots * G_{l}$ is simple then (re-ordering if necessary) we have either

(a) $G_{1}=G_{4}$ and $T_{2}=\cdots=T_{1}=K_{2}$

or

(b) $G_{1}=\theta_{r}$ and $T_{2}=\cdots T_{1}=K_{2}$.

Proof. First note that $G_{1} * \cdots * G_{l}$ is defined. By Lemma 4.1 each $T_{1}, i=1, \cdots, l$ is a star graph. Thus by Lemmas 4.5 and 4.6 each $G_{i}=\theta_{r}$ or $G_{i}=C_{4}$ for $i=1, \cdots, l$. Now it is easy to verify that for $r, s \geqq 2$ the graphs $C_{4} * \theta_{r}, \theta_{r} * \theta_{s}$ and $C_{4} * C_{4}$ are not simple. The only remaining possibilities are given in (a) and (b).

LEMMA 4.10. Let $G$ be a graph with a single cut point $x$ having no pendant vertices. If some cycle of $G$ does not contain $x$, then $G$ is not simple.

Proof. By Lemma 1.10 of [5] there is a transfer of $G$ which reduces the number of blocks of $G$ so that $G$ cannot be simple.

THEOREM 4.11. Let $G$ be a graph without pendant vertices and having a single cut point. Then $G$ is simple if and only if $G$ has one of the two following forms.

(a) $C_{4} * C_{3} * \cdots * C_{3}$

(b) $\theta_{r} * C_{3} * \cdots * C_{3}$.

Proof. To see that the graphs in (b) are simple it suffices to note that removing a point of degree $|V G|-1$ in (b) results in a graph of the form $K_{1, m} \cup m K_{2}$ which by Proposition 3.3 is simple, and so the original graphs, by Corollary 2.5, are simple. Also it is easy to verify that $C_{4} * C_{3}$ is simple and so the graphs in (a) are simple. 
For the converse note that by Lemma 4.10 the single cut point $x$ lies on each cycle of $G$ so that $G-x$ is a forest. Since there are no pendant vertices in $G$, the point $x$ must be adjacent to each point of degree one of $G-x$. This means that $G$ has to be of the form of the graph of Lemma 4.9 a 'product' of 1-cones and hence the result is true.

COROLlaRY 4.12. Let $G$ be a connected graph with $\delta(G) \geqq$ 2. Then $G$ is simple if and only if $G=H * C_{3} * \cdots * C_{3}$ where $H$ is either $C_{4}$ or $\theta_{r}$.

Proof. This result follows from Theorem 4.11.

Notice now that all simple graphs which are not blocks and which do not have pendant vertices have been characterized. We now turn our attention to the case when $G$ has pendant vertices. We need the following definition.

Definition. Let $G$ be a graph with $x, y \in V G$ and $e=$ $x y \in E G$. Then the subdivision of $G$ at $e, S_{e}(G)$, is the graph obtained by adding a new point $z$ to $V G$ and taking $(\{x z, y z\} \cup E G)-\{x y\}$ as the edge set.

REMARK. If a graph $G$ has a point of degree two that does not lie on a triangle, then it is clear that $G=S_{e}(H)$ for some $H$. It is also evident that if $e_{1}, e_{2} \in E G$, then $S_{e_{1}}(G)$ and $S_{e_{2}}(B)$ belong to the same degree sequence. This means that in order to show $S_{e}(G)$ is not simple we need only find some $e^{\prime} \in E G$ such that $S_{e}(G) \not \equiv S_{e^{\prime}}(G)$.

We now proceed to characterize all graphs $G$ for which $S_{e}(G)$ is simple.

LEMMA 4.13. Let $p$ be a positive integer, $p \geqq 6$. If $G$ is a regular graph of degree $r$, then $S_{e}(G)$ is simple if and only if $r=1$ or $r=p-1$.

Proof. If $r=1$, the result is clear, for then the graph has the form $n K_{2} \cup K_{1,2}$.

Case 1. Suppose $r$ is such that $2 \leqq r \leqq[p / 2]-1$. By Proposition 2.11 there are disconnected and connected graphs which are regular of degree $r$ and subdividing an edge in each of these graphs gives the result.

Case 2. Suppose $r$ is such that, $[p / 2] \leqq r \leqq p-3$. Since $p-1 \geqq$ 
$r \geqq 2$, it follows that $(r, \cdots, r)$ has two realizations $G_{1}$ and $G_{2}$ so that $G_{1}^{c}$ is a block and $G_{2}^{c}$ is disconnected. Further, $G_{2}^{c}$ has at least one component which is not complete since if each component of $G_{2}^{c}$ is complete it would follow that $G_{2}$ is a complete $k$-partite graph contradicting the fact that $G_{2}$ is regular of degree $r>[p / 2]$. From this it follows that there are $x, y \in V G$ such that $x$, and $y$ belong to a component of $G_{2}^{c}$ but $x y \notin E G_{2}^{c}$, and $x y \in E G_{2}$. But now the complement of $S_{x y}\left(G_{2}\right)$ has a cut point. Since the complement of $G_{1}$ is a block so is the complement of $S_{e}\left(G_{1}\right)$ where $e$ is any edge of $G$. Because $S_{x y}\left(G_{2}\right)$ and $S_{e}\left(G_{1}\right)$ belong to the same degree sequence, the result follows.

Case 3. $r=p-2$. In this case $\left[S_{e}(G)\right]^{c}=C_{5} * K_{3} * \cdots * K_{3}$ belongs to the same sequence as $C_{4} * C_{4} * K_{3} * \cdots * K_{3}$.

Case 4. $r=[p / 2]$. If $p=2 r$, note that Lemma 2.9 gives two realizations of $(r, \cdots, r)$, one with no triangles and one with a triangle. Now subdivide each one and in the latter choose an edge not on the triangle to subdivide. Hence the resulting subdivision graphs maintain the difference in the number of triangles. If $p=2 r+1$, then as in Lemma 2.8 we construct a graph $G$ as follows: Let $V G=$ $\left(x_{1}, \cdots, x_{r}\right\} \cup\left\{y_{1}, \cdots, y_{r}\right\} \cup\{z\}, \quad E g=\left\{x_{i} y_{i} \mid i \neq j, \quad 1 \leqq i, \quad j \leqq r\right\} \cup$ $\left\{x_{2 i-1} x_{2 i} \mid i=1, \cdots, r / 2\right\} \cup\left\{y_{i} z \mid i \in\{1, \cdots, r\}\right\}$. Then the graphs $S_{x_{1} y_{2}}(G)$ and $S_{x_{1} y_{3}}(G)$ have different numbers of triangles. Note that we can, as we have, assume $r$ even since $p$ is odd.

COROLlARY 4.14. Let $G$ be a connected regular graph of degree $r$ on p points. Then $S_{e} G$ is simple if and only if $G=K_{p}$ or $G=C_{4}$.

Proof. For $p \geqq 6$ the corollary follows by Lemma 4.13 and for $p \leqq 5$ the corollary follows by checking the six possibile cases.

THEOREM 4.15. Let $G$ be a connected graph, $e \in E G . \quad S_{e}(G)$ is simple if and only if $G$ is of one of the following forms:

(a) $C_{4}$

(b) $K_{1, n}$

(c) $C_{3} * \cdots * C_{3}$

(d) $K_{n}$.

Proof. Let $e, e^{\prime} \in E(G), \quad x, y, x^{\prime}, y^{\prime} \in V G \quad$ with $e=x y, \quad e^{\prime}=$ $x^{\prime} y^{\prime}$. We consider the two sets of numbers $S_{e}=\{\operatorname{deg} x, \operatorname{deg} y\}, S_{e^{\prime}}=$ $\left\{\operatorname{deg} x^{\prime}, \operatorname{deg} y^{\prime}\right\}$. Now if the latter two sets are distinct and at most two of the numbers $\operatorname{deg} x, \operatorname{deg} y, \operatorname{deg} x, \operatorname{deg} x^{\prime}$ are equal to two then $S_{e}(G) \neq S_{e^{\prime}}(G)$ since they have different adjacency relations. There remain two cases. 
Case 1. $S_{e}=S_{e^{\prime}}$ for all $e, e^{\prime} \in E G$. Here there are two subcases.

(i) $\operatorname{deg} x=\operatorname{deg} y$, or $G$ regular

(ii) $\operatorname{deg} x=\operatorname{deg} x^{\prime}, \operatorname{deg} y=\operatorname{deg} y^{\prime}$

In case (i) we can use Corollary 4.14 to obtain $G=K_{p}$ or $G=$ $C_{4}$. In case (ii) we have a bipartite graph. If $\operatorname{deg} y=1, G$ is a star Graph and $S_{e}(G)$ is simple since it is a Giap graph. Now assume $\operatorname{deg} y \geqq 2 ; \quad G$ is not regular implies $\operatorname{deg} x \neq \operatorname{deg} y, \quad$ say, $\operatorname{deg} x>$ $\operatorname{deg} y$. Notice that $G$ is bipartite and thus has no triangles. But because $\operatorname{deg} x>\operatorname{deg} y \geqq 2$ we can always make a transfer, $t$, to get a triangle and if $e_{0}$ is on an edge not on the triangle, then $S_{e_{0}}(t G)$ has a triangle, but $S_{e}(G)$ has no triangles.

Case 2. $S_{e} \neq S_{e^{\prime}}$ and $\operatorname{deg} x^{\prime}=\operatorname{deg} y^{\prime}=\operatorname{deg} y=2$ and $\operatorname{deg} x=k>$ 2. If there is another point $z$ of deg $k$ it cannot be adjacent to $x$ since then we be in the Case 1. Now $z$ and $x$ must have points of degree two separating them, so let $P$ be a path from $z$ to $x$. Note there is an edge $e_{0} \in E G$ not on $P$. Remove the points of degree two from $P$ (by "unsubdividing") and put them on $e_{0}$ (by repeated subdivision). This process yields a graph $H$ belonging to the same degree sequence but with points of degree $k$ adjacent. If $e_{1}$ is an edge incident with two points of degree $k$ and if $e_{2}$ is an edge incident with two points of degree two then $S_{e_{1}} H \not \equiv S_{e_{2}} H$. Thus we may assume that $x$ is the only point of degree $k$ so that it must be a cut-point of $G$ and is also the only cut point of $S_{e}(G)$. Hence, $S_{e}(G)$ is either $C_{4} * C_{3} * \cdots * C_{3}$ or $\theta_{r} * C_{3} * \cdots * C_{3}$. But the latter cannot be a subdivision graph since all points of degree two lie on a triangle. Thus $S_{e}(G)=C_{4} * C_{3} * \cdots * C_{3}$ so that $G=C_{3} * \cdots * C_{3}$. This gives the theorem.

Corollary 4.16. Let $G$ be a graph with a point of degree two that does not lie on a triangle with nontrivial components. $G$ is simple if and only if $G$ is $C_{4}, C_{5}, C_{4} * C_{3} * \cdots * C_{3},[2, m] G, S_{e}\left(K_{n}\right)$ or $s K_{2} \cup t[1,2] G$, $t \in\{0,1\}$.

Proof. The proof follows immediately from Theorem 4.15 and Proposition 4.1.

We now apply the latter results to characterize simple graphs with cut points and pendant vertices.

Proposition 4.17. If $G$ is a simple graph and $x$ a pendant vertex at $G$, then $G-x$ is also simple.

Proof. Let $x \in G, \operatorname{deg} x=1$. Let $S^{\prime}$ be the degree sequence of $G-x$ and suppose that $H_{1}$ and $H_{2}$ belong to $S^{\prime}$. We can assume that $V G-\{x\}=V H_{1}=V H_{2}$, and that $H_{1}=t H_{2}$ so that the degree of any 
point in $V G-\{x\}$ is the same in $H_{1}$ as in $H_{2}$. Let $x^{\prime} \in v_{G}$ be the point to which $x$ is adjacent in $G$, let $G_{1}, G_{2}$ be the graphs obtained from $H_{1}, H_{2}$, respectively, by attaching $x$ to $x^{\prime}$. Then $G$ simple implies $G_{1} \cong$ $G_{2}$. Hence, let $F: V G_{1} \rightarrow V G_{2}$ be the isomorphism. If $f\left(x^{\prime}\right)=x^{\prime}$, then $f(x)$ is $x$ or some other point of degree one adjacent to $x^{\prime}$. If $f(x)=x$, then $\left.f\right|_{V H_{1}}$ is an isomorphism of $H_{1}$ onto $H_{2}$. Let $N_{x^{\prime}} \cap S_{1}$ denote the set of points of degree one adjacent to $x^{\prime}$. Then $f$ permutes the points of this set. So let $f^{*}: V G \rightarrow V G$ be such that $f(y)=y$ for $y \in V G-\left(N_{x^{\prime}} \cap S_{1}\right)$ and on $N_{x^{\prime}} \cap S_{1}, f^{*}$ is the inverse of $f$. Then $f * f: V G \rightarrow V G$ is an isomorphism of $G$ such that $(f * f)(x)=x$, so that $\left.f * f\right|_{V H}$ is an isomorphism. Thus, if $f\left(x^{\prime}\right)=x^{\prime}$, we are done. So suppose $y^{\prime}, z^{\prime}$ such that $f\left(y^{\prime}\right)=x^{\prime}, f\left(x^{\prime}\right)=z^{\prime}$. Since $f$ is an isomorphism, each of $x^{\prime}, y^{\prime}$ and $z^{\prime}$ are adjacent to the same number of pendant vertices. Now if $t \in V G-S_{1}$ is adjacent to any of $x^{\prime}, y^{\prime}, z^{\prime}$ it must be to all. To see this latter suppose the contrary, that $w x^{\prime} \in E G$, $w y^{\prime} \notin E G$ and $y^{\prime \prime}$ a point of degree one adjacent to $y^{\prime}$. Then the transfer $t$ of $w x^{\prime}$ and $y^{\prime} y^{\prime \prime}$ for $w y^{\prime}$ and $x^{\prime} y^{\prime \prime}$ is defined and $G \neq t G$ since $t G$ has fewer vertices adjacent to. $\left|N_{x^{\prime}} \cap S_{1}\right|$ points of degree one then $G$ does, contradicting the simplicity of $G$. Because of this latter property, the function $f^{\prime}$ defined by:

$$
\begin{aligned}
& f^{\prime}(z)=z, z \in N_{x^{\prime}} \cap S_{1} \\
& f^{\prime}\left(x^{\prime}\right)=x^{\prime} \\
& f^{\prime}\left(y^{\prime}\right)=z^{\prime}=f\left(f\left(y^{\prime}\right)\right) \\
& f^{\prime}(s)=f(f(s)) \quad \text { if } \quad s y^{\prime} \in E G \\
& f^{\prime}(z)=f(z), z \quad V G-\left\{\left(\left(N_{x^{\prime}} \cup N_{y^{\prime}} \cup N_{z^{\prime}}\right) \cap S_{1}\right) \cup\left\{y^{\prime}\right\}\right\}
\end{aligned}
$$

is an isomorphism mapping $x^{\prime}$ to itself so that be the first part of the proof we have $H_{1} \cong H_{2}$.

COROLlary 4.18. Let $G$ be a simple graph and let $S_{1}$ be the set of pendant vertices of $G$. Then for any subset $S$ of $S_{1},\langle V G-S\rangle$ is simple.

Proof. To obtain the corollary, apply the previous proposition repeatedly $|S|$ times.

Proposition 4.19. Let $G$ be a simple graph with nontrivial components and let $S_{1} \neq V \dot{G}$ be the set of pendant vertices of $G$ then $<V G-S_{1}>$ is either a block, a $K_{2}$, or is a graph of the form 


$$
C_{4} * C_{3} * \cdots * C_{3} \text { or } \theta_{r} * C_{3} * \cdots * C_{3} \text {. }
$$

Proof. If $G$ is not connected, and $S_{1} \neq V G$ then $<V G-S_{1}>$ simple and from Proposition 4.1 it is clear that $<V G-S_{1}>$ is $K_{2}$.

Now assume that $\left\langle V G-S_{1}\right\rangle$ is connected. If it is not a block, it has a cut point. Now $<V G-S_{1}>$ has no pendant vertices or $\left\langle V G-S_{1}\right\rangle=K_{2}$. To see this, let $x \in\left\langle V G-S_{1}\right\rangle$ be a pendant vertex. Since $x \notin S_{1}$ in $G$, we have $\operatorname{deg} x \geqq 1$ so that $x$ was adjacent to some $y \in S_{1}$. Then $<\left(V G-S_{1}\right) \cup\{y\}>$ has a point of degree two not lying on any triangle (namely $x$ ) and is connected. Hence < $\left(V G-S_{1}\right) \cup\{y\}>$ is a subdivision graph, so that by Corollary 4.16 we have $<\left(V G-S_{1}\right) \cup\{y\}>=[2, m] G$. But $G$ is simple, and the difference between $G$ and $<\left(V G-S_{1}\right) \cup\{y\}>$ consists of points of degree one, so $G$ is a Giap graph and hence, $\langle V G-S\rangle=K_{2}$.

Now assume that $G$ is not a Giap graph, so that $\delta\left(<V G-S_{1}>\right) \geqq$ 2 , and $\left\langle V G-S_{1}\right\rangle$ is not a block. By Corollary $\left.4.12<V G-S_{1}\right\rangle$ is $H * C_{3} * \cdots * C_{3}$ where $H=\theta_{r}$ or $C_{4}$. This gives the result.

Corollary 4.20. Let $G$ be connected simple graph with pendant vertices. Then $G$ has one of the following forms:

(a) $[n, m] G$

(b) $H * C_{3} * \cdots * C_{3} * K_{2} * \cdots * K_{2}$ where $H=\theta_{r}$ or $H=C_{4}$

(c) $<V G-S_{1}>$ is a simple block, where $S_{1}$ is the set of pendant vertices of $G$.

Proof. This corollary follows from Proposition 4.19 and the fact that the only way to add a pendant vertex to a graph of the form $H * C_{3} * \cdots C_{3}$ if we want to preserve simplicity is at the vertex of highest degree.

Theorem 4.21. Summary. Let $G$ be a simple graph which is not a block. The $G$ has one of the following forms.

(a) $s K_{2} \cup t[n, m] G$ where $s$ is a nonnegative integer $t \in\{0,1\}$ and $s \neq 0$ implies $n=1$ or $m=1$.

(b) $H * C_{3} * \cdots * C_{3} * K_{2} * \cdots * K_{2}$ where $H=C_{4}$ or $H=\theta_{r}$.

(c) $<V G-S_{1}>$ is a simple block where $S_{1}$ is the set of pendant vertices of $G, S_{1} \neq V G$.

(d) the union of one graph from (a), (b) or (c) with any number of trivial graphs.

REMARK. In view of Theorem 4.21 it follows that any simple graph $G$ which is separable with $\delta(G) \geqq 2$, must satisfy one of the following degree sequences 

(a) $(m, 2, \cdots, 2)=m 2^{m}$ or $m 2^{m-1}$
(b) $(m, r+1,2, \cdots, 2)=m^{1}(r+1)^{1} 2^{m}$.

\section{REFERENCES}

1. Gary Chartrand and Mehdi Behzad, Introduction to the Theory of Graphs, Allyn and Bacon (1971).

2. Fulkerson, Hoffman and McAndrew, Some Properties of Graphs with Multiple Edges, Canad. J. Math. 17 N-1. (1965), 166-177.

3. S. L. Hakimi, On the Realizability of a Set of Integers as Degrees at the Vertices of a Graph, II Uniqueness, S/AM J. App. Math. (11) No. 1, (March 1963), 135-147.

4. Frank Harary, Graph Theory, Addison Wesley (1969).

5. R. H. Johnson, Graphical Sequences, Ph.D. Thesis, Virginia Polytechnic Institute and State University.

6. J. K. Senior, Partitions and their Representative Groups, Amer. J. Math., 73 (1951), 663-689.

Received September 28, 1973 and in revised form May 6, 1974.

California State College, Sonoma 



\section{Pacific Journal of Mathematics}

\section{Vol. 56, No. $1 \quad$ November, 1975}

Shimshon A. Amitsur, Central embeddings in semi-simple rings .......... 1

David Marion Arnold and Charles Estep Murley, Abelian groups, A, such

that $\operatorname{Hom}(A,---)$ preserves direct sums of copies of $A \ldots \ldots \ldots .$.

Martin Bartelt, An integral representation for strictly continuous linear

operators ................................... 21

Richard G. Burton, Fractional elements in multiplicative lattices......... 35

James Alan Cochran, Growth estimates for the singular values of

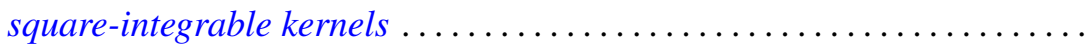

C. Martin Edwards and Peter John Stacey, On group algebras of central

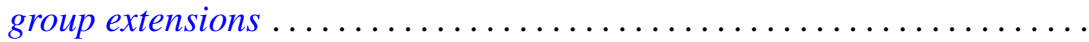

Peter Fletcher and Pei Liu, Topologies compatible with homeomorphism

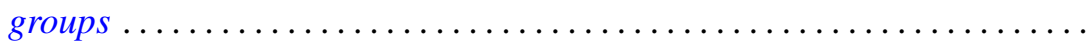

George Gasper, Jr., Products of terminating ${ }_{3} F_{2}(1)$ series ............ 87

Leon Gerber, The orthocentric simplex as an extreme simplex ............

Burrell Washington Helton, A product integral solution of a Riccati

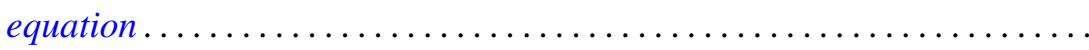

Melvyn W. Jeter, On the extremal elements of the convex cone of

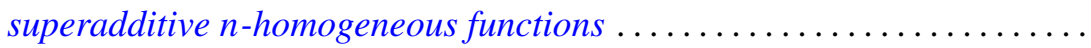

R. H. Johnson, Simple separable graphs .

Margaret Humm Kleinfeld, More on a generalization of commutative and

alternative rings. . .

A. Y. W. Lau, The boundary of a semilattice on an $n$-cell.

Robert F. Lax, The local rigidity of the moduli scheme for curves ...

Glenn Richard Luecke, A note on quasidiagonal and quasitriangular

operators .

Paul Milnes, On the extension of continuous and almost periodic functions

Hidegoro Nakano and Kazumi Nakano, Connector theory.

James Michael Osterburg, Completely outer Galois theory of perfect rings ..................................

Lavon Barry Page, Compact Hankel operators and the F. and M. Riesz



Joseph E. Quinn, Intermediate Riesz spaces..................... 225

Shlomo Vinner, Model-completeness in a first order language with a generalized quantifier.

Jorge Viola-Prioli, On absolutely torsion-free rings ..........

Philip William Walker, A note on differential equations with all solutions of integrable-square............................

Stephen Jeffrey Willson, Equivariant maps between representation 